\title{
Estratégias relacionadas à conservação ambiental em reservas particulares no Mato Grosso do Sul, Brasil
}

\author{
Strategies related to environmental conservation in private \\ reserves in Mato Grosso do Sul, Brazil
}

\author{
Marta Regina da Silva Meloa \\ Celso Correia Souzab \\ Neiva Maria Robaldo Guedes ${ }^{c}$
}

\begin{abstract}
${ }^{a}$ Mestre em Meio Ambiente e Desenvolvimento Regional do Programa de Pós-Graduação da Universidade Anhanguera Uniderp, Bolsista da Coordenação de Aperfeiçoamento de Pessoal de Nível Superior, Capes, Campo Grande, MS, Brasil.

End. Eletrônico: martamelors@gmail.com
\end{abstract}

${ }^{b}$ Professor Doutor do Programa de Pós-Graduação em Meio Ambiente e Desenvolvimento Regional da Universidade Anhanguera Uniderp, Campo Grande, MS, Brasil. End. Eletrônico: csouza939@gmail.com

cProfessora Doutora do Programa de Pós-Graduação em Meio Ambiente e Desenvolvimento Regional da Universidade Anhanguera Uniderp, Campo Grande, MS, Brasil. End. Eletrônico: guedesneiva@gmail.com

doi:10.18472/SustDeb.v9n2.2018.26669

\section{RESUMO}

Iniciativas voltadas para conservação de Áreas Protegidas (APs) se destacam como ações essenciais para a proteção do ambiente natural e seus elementos. $O$ estudo tem como objetivo analisar estratégias relacionadas à conservação ambiental nas Reservas Particulares do Patrimônio Natural (RPPNs) Quinta do Sol e Vale do Bugio, Mato Grosso do Sul. A pesquisa foi conduzida a partir de uma abordagem qualiquantitativa e multidisciplinar. Verificou-se também 12 variáveis como indicadores de sustentabilidade ambiental, compatíveis com as duas reservas. A propagação das estratégias relacionadas à conservação ambiental utilizadas por essas reservas pode contribuir de forma significativa para a expansão de novas áreas protegidas em regiões importantes, como é o caso da Serra de Maracaju, área prioritária para conservação da biodiversidade.

Palavras-chave: Áreas Protegidas; Conservação; Gestão Sustentável; Indicadores Ambientais. 


\begin{abstract}
Initiatives aimed at the conservation of Protected Areas (PAs) stand out as essential actions for the protection of the natural environment and its elements. This study aims to analyze strategies related to environmental conservation in the Private Reserves of Natural Heritage (RPPNs) Quinta do Sol and Vale do Bugio, in the Mato Grosso do Sul State. The research was conducted with a quali-quantitative and multidisciplinary approach. Twelve variables compatible with the two reserves were verified as environmental sustainability indicators. The propagation of environmental conservation strategies used by these reserves can contribute significantly to the expansion of new protected areas in important regions, as is the case of Serra de Maracaju, a priority area for biodiversity conservation.
\end{abstract}

Keywords: Protected Areas; Conservation; Sustainable Management; Environmental Indicators.

\title{
1 INTRODUÇÃO
}

A atuação e as alterações realizadas pelo homem no planeta causam perdas irreversíveis à biodiversidade, por isso diferentes estratégias têm sido implantadas a fim de mitigar as ações do homem que prejudicam os ambientes naturais (LE SAOUT et al., 2013). O empenho para a conservação da natureza concentra-se muitas vezes sobre a criação de diferentes categorias de áreas protegidas e surge como estratégia viável para abrigar e proteger a biodiversidade e os recursos naturais (CHAPE et al., 2005; WATSON et al., 2014).

O Brasil detém uma das mais ricas biodiversidades do planeta em seus distintos domínios morfoclimáticos. Essa abundante variedade de vida se traduz em mais de $13 \%$ do número total de espécies da biota mundial (LEWINSOHN; PRADO, 2005), considerada a principal nação em biodiversidade do mundo. Devido a sua extensão territorial, o Brasil comporta um vasto mosaico das principais paisagens do mundo tropical (AB'SABER, 2012), representadas em seus domínios morfoclimáticos e fitogeográficos. Entre os domínios, o Cerrado brasileiro constitui a savana mais rica em biodiversidade da América do Sul e o bioma mais modificado pelo homem (OLIVEIRA et al., 2015; CARDOSO et al., 2015). Junto com a Mata Atlântica integram as 25 ecorregiões mais ricas e ameaçadas do planeta, os hotspots (MYERS et al., 2000; MAGANHOTTO et al., 2014).

Desse modo, com a finalidade de garantir a conservação dos ecossistemas, da diversidade biológica e recursos naturais, foi estabelecido o Sistema Nacional de Unidades de Conservação da Natureza (Snuc) - Lei federal 9.985/2000, que define as Unidades de Conservação (UCs) como áreas naturais passíveis de proteção por suas características relevantes (BRASIL, 2000).

Verifica-se um interesse crescente no mundo para o uso e conservação da biodiversidade em propriedades privadas (PEGAS; CASTLEY, 2015). Nesse sentido, foram estabelecidas no Brasil as Reservas Particulares do Patrimônio Natural (RPPNs) com o intuito de promover a conservação da biodiversidade, educação, desenvolvimento de pesquisas científicas e atividades de turismo sustentável (BRASIL, 2000; DOUROJEANNI; PÁDUA, 2013). Define-se RPPN como uma área privada, gravada com perpetuidade e averbada na escritura do imóvel (CARDOSO et al., 2015). Essas áreas constituem uma parte vital para o sistema brasileiro de áreas protegidas, o qual estabeleceu as Unidades de Conservação (CROUZEILLES et al., 2012; MORAES et al., 2014).

Dourojeanni e Pádua (2013) ressaltam que se existe uma iniciativa para a conservação da biodiversidade que vem dando certo no Brasil, é o estabelecimento de Reservas Particulares do Patrimônio Natural. A conservação em terras privadas se configura pelo seu importante papel na promoção de conservação da natureza (KAMAL; GRODZINSKA-JURCZAKA; BROWN, 2015) e representa relevante mecanismo no processo de conservação ambiental, especialmente por ser uma iniciativa voluntária.

O Brasil possui 1.362 RPPNs que correspondem a 759.485,25 hectares de áreas protegidas, entre as quais, 51 dessas reservas se encontram no Mato Grosso do Sul e abrangem um total de 146.681,31 ha, distribuídas em 24 municípios (ALVES et al., 2016).

O Mato Grosso do Sul compõe uma complexa 
combinação de ambientes naturais com abundante diversidade biológica em seus diferentes ecossistemas e abriga três significativos domínios morfoclimáticos: o Cerrado, a Mata Atlântica e o Pantanal (AB'SABER, 2012). Foi o primeiro estado brasileiro a promulgar legislação específica para as Reservas Particulares do Patrimônio Natural (Decreto Estadual no 7.251, de 16 de junho de 1993). Devido a esse fato, juntamente com o interesse de alguns proprietários, foi classificado como um dos estados brasileiros com maior número de áreas protegidas privadas (PELLIN; RANIERI, 2009).

A utilização de indicadores de sustentabilidade ambiental em UCs pode representar uma ferramenta orientadora para a tomada de decisões, tanto no que se refere à gestão, quanto no fortalecimento das interfaces da sustentabilidade (ELBAKIDZE et al., 2013; FILETTO; MACEDO, 2015), haja vista que a eficiência da sustentabilidade está na possibilidade em envolver estratégias que minimizem danos causados pelo homem ao meio ambiente (VAN BELLEN, 2006; MORELLI, 2011).

Dessa forma, a utilização da percepção ambiental direcionada a essas áreas pode elevar à compreensão das inter-relações entre o homem e o ambiente, bem como para o planejamento das ações praticadas quanto à utilização racional dos recursos naturais (LUCENA; FREIRE, 2011). Conhecer e compreender o impacto dessas ações pode ser um caminho para o aprimoramento de uma gestão sustentável em áreas protegidas, além de fortalecer experiências relacionadas às estratégias para a proteção e a conservação da natureza.

O presente estudo tem como objetivo analisar estratégias relacionadas à conservação ambiental nas Reservas Particulares do Patrimônio Natural Estância Ambiental Quinta do Sol e Reserva Ecológica Vale do Bugio, no município de Corguinho, Mato Grosso do Sul. Especificamente, avaliar a percepção ambiental dos visitantes e apresentar indicadores de sustentabilidade ambiental relacionados às estratégias voltadas para a conservação ambiental.

\section{METODOLOGIA}

\subsection{CARACTERIZAÇÃO DAS ÁREAS DE ESTUDO}

As RPPNs Estância Ambiental Quinta do Sol e a Reserva Ecológica Vale do Bugio estão localizadas em Corguinho, Mato Grosso do Sul. Ambas representativas do Cerrado. Situam-se na Serra de Maracaju, cenário de relevante beleza cênica que se estende no sentido norte-sul por todo o estado de Mato Grosso do Sul, e atua como um divisor de águas entre as bacias hidrográficas do Alto Rio Paraguai e do Rio Paraná.

\subsection{ESTÂNCIA AMBIENTAL QUINTA DO SOL}

Criada pela Resolução Estadual no 16/2011, a Estância Ambiental Quinta do Sol possui uma área de 12,69 ha, constituída por tipos vegetacionais do Cerrado, com grande parte recuperada após o estabelecimento da UC. Localiza-se nas coordenadas geográficas $55^{\circ} 14^{\prime} 37^{\prime \prime} \mathrm{W}, 19^{\circ} 43^{\prime} 60^{\prime \prime} \mathrm{S}$, no distrito de Taboco, município de Corguinho (Figura 1). 


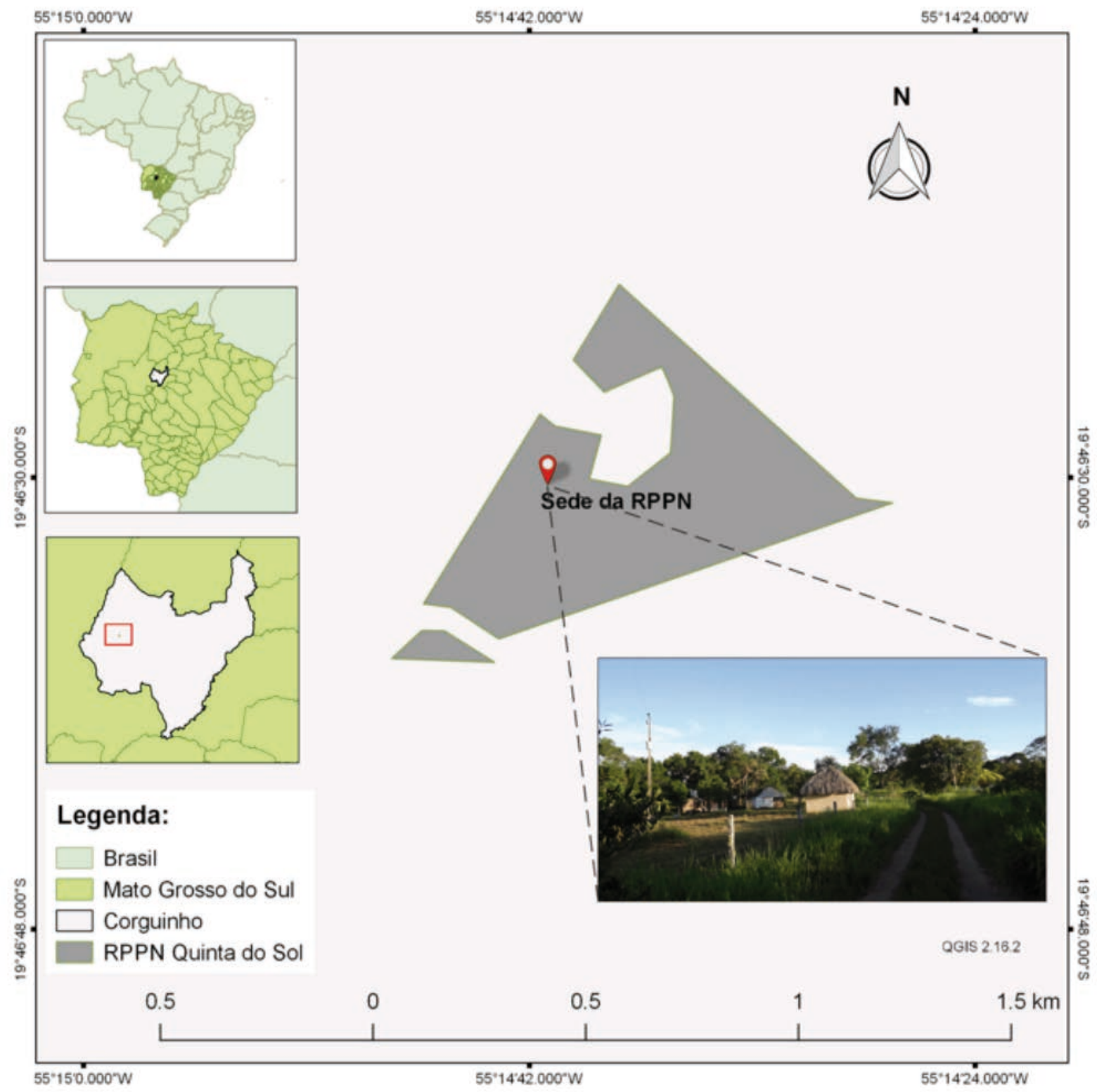

Figura 1 - Mapa de localização da RPPN Quinta do Sol, no município de Corguinho, Mato Grosso do Sul, Brasil.

Em parceria com a Wildlife Conservation Society (WCS Brasil), a Estância Ambiental Quinta do Sol contribui para a conservação da biodiversidade com ações pautadas na educação ambiental, aplicadas a projetos de conservação da vida silvestre. Esses projetos de conservação investem na sensibilização da população local, uso sustentável da terra e no desenvolvimento de pesquisa científica.

Um dos projetos de grande visibilidade internacional é o Projeto Queixada, cujo objetivo é o de identificar áreas de deslocamento da espécie e analisar quais os componentes da sua dieta alimentar. Dentro dessa perspectiva, a espécie Tayassu pecari é considerada importante indicador da paisagem, das condições ambientais e desempenha importantes funções ecológicas no ecossistema (KEUROGHLIAN et al., 2014). Ainda como estratégias de conservação, são desenvolvidos cursos de capacitação com a utilização de técnicas de bioconstrução e permacultura, restauração ambiental, Biologia de Campo, entre outros relacionados à proteção do ambiente natural. As atividades práticas desenvolvidas têm por finalidade proporcionar a troca de experiências entre alunos e docentes de diferentes instituições de ensino que utilizam a área para ampliar o conhecimento. 


\subsection{RESERVA ECOLÓGICA VALE DO BUGIO}

Em 2003 foi publicada pela Secretaria de Estado e Meio Ambiente (Sema) a deliberação de no 07, que criou a RPPN Vale do Bugio, com área de $81,75 \mathrm{ha}$, situada nas coordenadas geográficas $55^{\circ} 04^{\prime} 14.41^{\prime \prime}$ W e $19^{\circ} 56^{\prime} 19.46^{\prime \prime} \mathrm{S}$, no interior de uma furna formada pela cuesta dos arenitos de Botucatu (Figura 2).

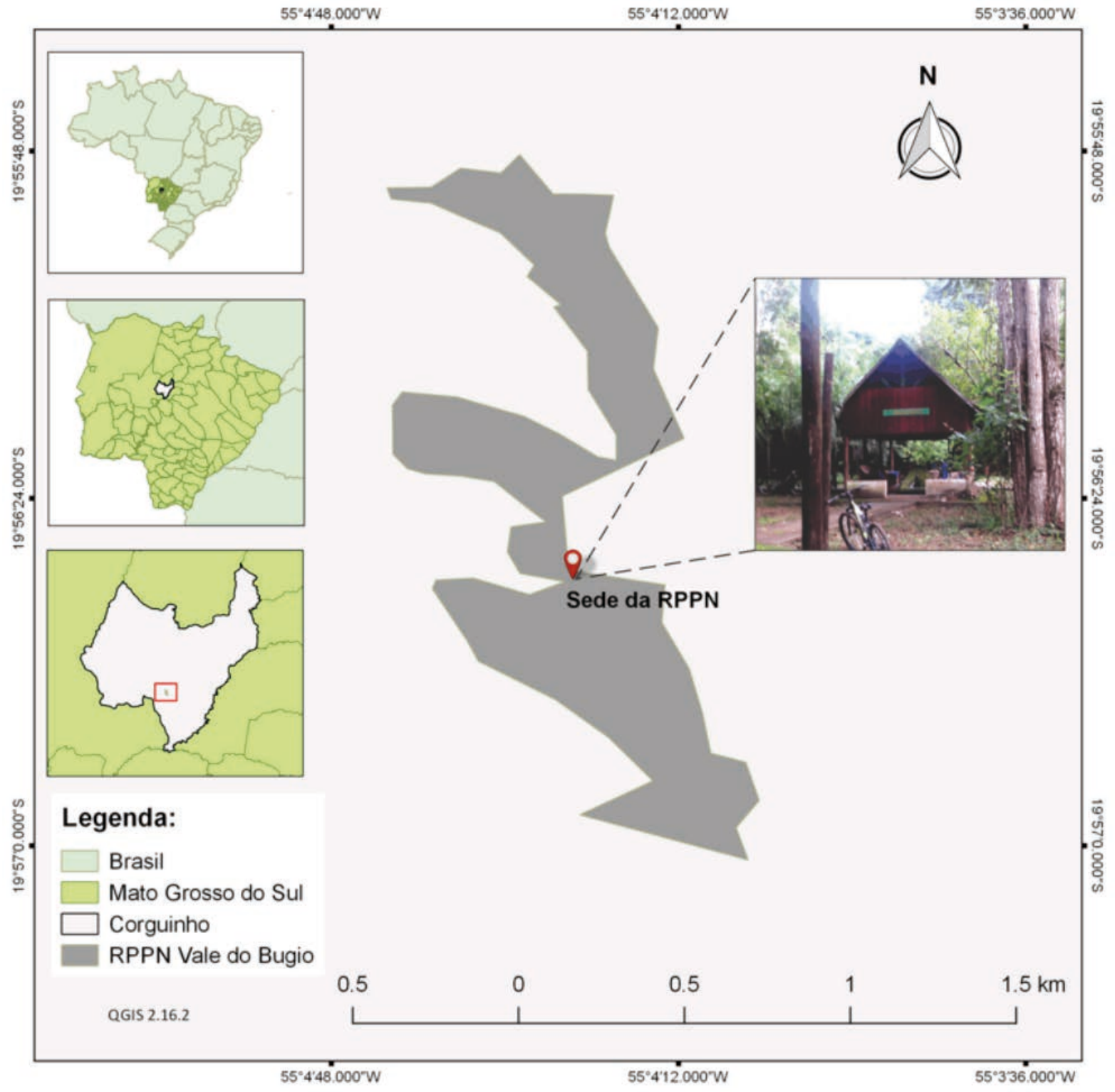

Figura 2 - Mapa de localização da RPPN Vale do Bugio, no município de Corguinho, Mato Grosso do Sul, Brasil.

Fonte: Elaborada pelo autor.

Nessa área são desenvolvidas diferentes atividades, tais como: cursos de Biologia de Campo e acampamentos de férias para escolares; cursos diversos e ações voltadas para a Educação Ambiental; turismo científico e de vida silvestre; turismo de observação de aves; e a pesquisa científica. A Reserva ainda representa o local de reprodução da espécie Spizaetus ornatus, o gavião-de-penacho, onde é desenvolvido um projeto que tem como principal objetivo monitorar o sucesso reprodutivo da espécie na região da Serra de Maracaju, planalto de entorno do Pantanal. 


\subsection{MÉTODOS DE ANÁLISE}

Os procedimentos metodológicos adotados para a elaboração desta pesquisa, a fim de analisar as estratégias relacionadas à conservação ambiental nas RPPNs Quinta do Sol e Vale do Bugio, foram conduzidos sob uma abordagem mista e multidisciplinar (BURSZTYN, 2004), uma vez que os métodos mistos podem ser aplicados como um procedimento de coleta, análise e combinação de técnicas quantitativas e qualitativas em um mesmo desenho de pesquisa (CRESWELL; PLANO CLARK, 2011). Ainda, para apresentação e sistematização de indicadores de sustentabilidade, foram realizados levantamentos bibliográficos, análise documental e observações in loco.

Para avaliar a percepção dos visitantes sobre o papel dessas UCs e seu impacto na conservação ambiental, foram aplicados 103 questionários para a população visitante, aprovados pelo Comitê de Ética em Pesquisa conforme o no 1.361.821/2015, sendo 51 na Reserva Quinta do Sol e 52 na Vale do Bugio.

Em relação ao grau de satisfação do pesquisado ao visitar a RPPN, foi empregada uma escala de Likert, com variação de 1 a 10, onde 1 = menor grau de satisfação, até 10 = maior grau de satisfação. A escala proporciona capturar variáveis qualitativas e as transformam em quantitativas.

Com a utilização do software Sphinx Léxica 5.0, os formulários de pesquisa foram tabulados e utilizado o método de análise univariada, a fim de examinar uma variável por vez, e bivariada, para verificar as relações existentes entre duas variáveis.

Os dados coletados a partir da percepção ambiental dos visitantes das duas RPPNs foram compilados em tabelas de contingência para analisar as relações entre as variáveis. $O$ teste Qui-Quadrado $\left(X^{2}\right)$ foi utilizado para o grau de significância, onde $p$ - valor $<0,01$ (altamente significativo) e $p$ - valor $<0,05$ (significativo).

Com o propósito de destacar a importância das estratégias de conservação desenvolvidas pelas RPPNs e para apontar ações que refletem indicadores ambientais, foram identificados os parâmetros ambientais de sustentabilidade das RPPNs e em seguida foram agrupados a partir de características comuns com base na metodologia de Marinelli e Maltez (2010). Para analisar os indicadores de sustentabilidade ambiental nas RPPNs Quinta do Sol e Vale do Bugio, foram realizadas adaptações baseadas na metodologia utilizada por Marangon et al. (2004), que permitem uma dimensão qualitativa de ocorrência em níveis Altos (A), Médios (M) e Baixos (B). Para estabelecer a divisão dos níveis, considera-se uma escala de 1 a 100 (da menor para a maior incidência verificada) e divide-se por três (relativos aos níveis $A, M, B$ ), os quais resultam na seguinte padronização:

- Alto - corresponde a uma ocorrência compreendida entre $66,67 \%$ e $100 \%$;

- Médio - corresponde a uma ocorrência compreendida entre 33,34\% e 66,66\%; e

- Baixo - corresponde a uma ocorrência compreendida entre $1 \%$ e 33,33\%.

Embora os indicadores ambientais de modo geral não apresentem valores absolutos, tem-se uma noção do que é aceitável para a sustentabilidade (MOLDAN; JANOUŠKOVÁ; HÁK, 2012). Entretanto, podem ser utilizados na aplicação de estratégias voltadas para a conservação.

\section{RESULTADOS E DISCUSSÃO}

Observou-se que durante o período de amostragem, 39,8\% dos visitantes das reservas foram compostos por homens e $60,2 \%$ por mulheres, dos quais as idades variaram entre 15 e mais de 60 anos, com destaque para a faixa etária de 26 a 36 anos, que representou $31,1 \%$ dos participantes da pesquisa.

Sobre o grau de escolaridade dos visitantes, o nível superior apresentou maior percentual, com índice de $47,6 \%$. Portanto, deve-se ter em conta que os níveis de percepção ambiental dos grupos humanos possuem bagagens experienciais distintas devido a elementos, como cultura, faixa etária, gênero, nível socioeconômico, entre outros, que revelam percepções sob diversas formas (GONÇALVES; HOEFFEL, 2012). 


\subsection{PERCEPÇÃO AMBIENTAL DOS VISITANTES}

Para investigar a percepção dos visitantes sobre o papel das RPPNs e seu impacto para a conservação ambiental, foi questionado "o que o motivou a visitar a RPPN: Pesquisa Científica, Educação Ambiental ou o Ecoturismo" (Figura 3).

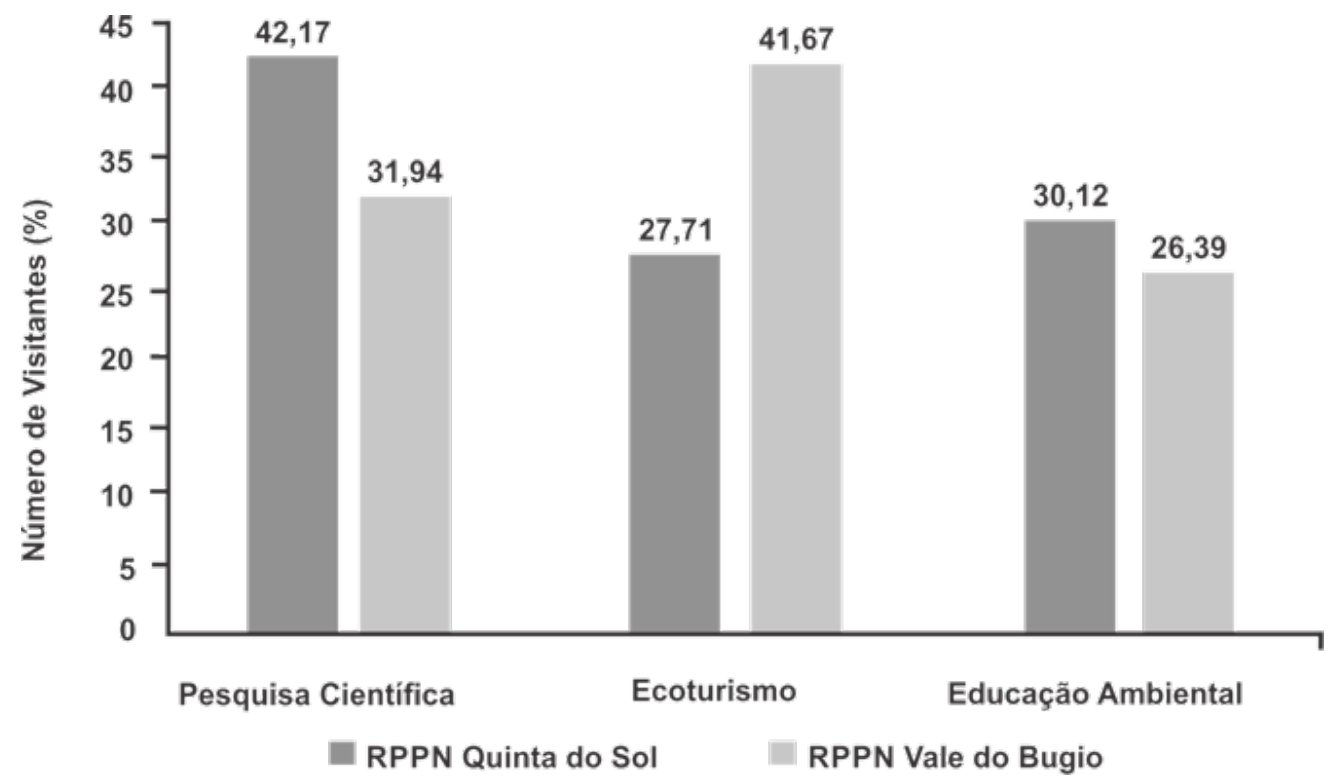

Figura 3 - Relevância de como as atividades de Pesquisa Científica, Ecoturismo e Educação Ambiental influenciaram as visitas nas RPPNs Quinta do Sol e Vale do Bugio, no município de Corguinho, Mato Grosso do Sul.

Fonte: Dados da pesquisa (2016).

Os índices apresentados para a motivação das visitas nas RPPNs Quinta do Sol e Vale do Bugio tiveram como destaque a pesquisa científica e o ecoturismo, devido à vocação dessas reservas para o desenvolvimento dessas atividades.

Assim, a reserva Quinta do Sol despontou para a pesquisa científica como atividade motivadora. Isso ocorre porque o público visitante, em grande parte, são alunos de Instituições de Ensino Superior e professores desenvolvedores de pesquisas no interior da reserva e entorno, além do turismo científico.

Os visitantes da reserva Vale do Bugio apresentaram como elemento motivador o ecoturismo, decorrente de atividades de cicloturismo e do turismo de observação de aves e da vida silvestre.

A respeito da questão "qual o grau de satisfação ao visitar a RPPN", os desvios padrão variaram de 12,72, para a RPPN Vale do Bugio, e 13,48 para a RPPN Quinta do Sol, com a indicação de que há homogeneidade na dispersão das informações analisadas e revelaram que o grau de satisfação dos visitantes das RPPNs foi altamente significativo (Tabela 1).

Tabela 1 - Representação do grau de satisfação dos visitantes de duas RPPNs

\begin{tabular}{ccccc}
\hline Grau de satisfação ao visitar a RPPN & N & Média & DP & CV \\
\hline Vale do Bugio & 51 & 9,35 & 1,19 & 12,72 \\
Quinta do Sol & 52 & 8,90 & 1,20 & 13,48 \\
\hline
\end{tabular}

1 = menor grau de satisfação e 10 = maior grau de satisfação

Fonte: Dados da pesquisa (2016). 
As informações apresentadas se enquadram nas Diretrizes para visitação em Unidades de Conservação elaboradas pelo Ministério do Meio Ambiente (2008), as quais sinalizam que o planejamento e a gestão da visitação devem considerar múltiplas formas de organização, com o dever de procurar satisfazer as expectativas dos visitantes no que diz respeito à qualidade e variedade das experiências, segurança e necessidade de conhecimento.

Ao questionar "de que forma as RPPNs podem contribuir para o setor econômico do país", 75,6\% dos visitantes atribuíram ao ecoturismo como uma atividade que contribui fortemente para a economia do país (Tabela 2).

Tabela 2 - Nível de contribuição das RPPNs Quinta do Sol e Vale do Bugio para a economia do país, segundo a percepção dos entrevistados

\begin{tabular}{lccc}
\hline \multicolumn{1}{c}{ Contribuição para o setor econômico } & N & \% \\
\hline Promover o ecoturismo aliado à educação ambiental & 75 & 75,6 \\
Promover cursos de formação e capacitação & 12 & 11,7 \\
Comercialização de projetos de sequestro e crédito de carbono & 12 & 11,7 \\
Fornecimento de cotas de reserva para outros proprietários & 4 & 1,0 \\
\hline
\end{tabular}

Fonte: Dados da pesquisa (2016).

O ecoturismo, além de caracterizar-se como um segmento rentável, se sobressai como veículo de educação ambiental, o que o torna uma das formas para a conscientização e disseminação de práticas de conservação ambiental (KLEIN et al., 2011). Além de figurar como estratégia de conservação, o ecoturismo apoia o desenvolvimento socioeconômico da região. Para isso, é fundamental que as práticas das atividades ecoturísticas aliadas à educação ambiental ocorram continuamente, tendo em vista que a educação ambiental é um processo que visa o desenvolvimento de cidadãos responsáveis e empenhados com a proteção e melhoria do meio ambiente (SAKELLARI; SKANAVIS, 2013).

Foi questionado "quem é o principal responsável pela conservação da natureza e de seus recursos". Como resposta, para um índice de $91,3 \%$ dos visitantes entrevistados, tanto o governo como a sociedade em geral são responsáveis diretos pela conservação ambiental e dos recursos naturais. É oportuno enfatizar que a sociedade passa por transformações e tem entendido que o meio ambiente deve ser protegido, em consequência de que o principal responsável pela degradação ambiental é o homem (NEIMAN, 2008).

Questionou-se aos visitantes se eles consideravam que "o contato com a natureza melhora as relações humanas?" Ao responderem essa questão, todos foram unânimes ao sinalizarem "sim" (100\%). Ainda para complementar esse questionamento, perguntou-se "em quais aspectos o contato com a natureza melhora as relações humanas?" Entre os participantes, $71,8 \%$ enfatizaram que "aumenta o sentido de pertencimento à natureza".

Compreende-se que a percepção dos visitantes das RPPNs Quinta do Sol e Vale do Bugio, para tal questionamento, corrobora a hipótese da Biofilia criada por Wilson (1984), na qual o autor especifica que a inclinação humana de exaltar a natureza e procurar um contato íntimo com ela é a manifestação de uma necessidade biológica, pois nossa composição genética se estruturou em função de nossa vida em ambientes naturais e não nos ambientes artificiais. O contato com a natureza transmite sensação de bem-estar, e seus elementos quando conservados propiciam benefícios ambientais. Eles resultam da regulação dos processos naturais, como a qualidade do ar, regulação do clima, regulação e purificação da água, controle de erosão e outros atributos considerados serviços ambientais (CONSTANZA et al., 1997). Desse modo, o vínculo entre o homem e a natureza pode ser a chave para a conservação ambiental. 
Por viabilizar a relação entre duas variáveis e determinar se as diferenças foram estatisticamente significativas, o uso da análise bivariada no estudo permitiu apresentar os resultados conforme o grau de significância da amostra investigada (Tabela 3).

Tabela 3 - Análise do cruzamento das variáveis relativas à percepção ambiental dos visitantes das RPPNs Quinta do Sol e Vale do Bugio

\begin{tabular}{lcc}
\hline \multicolumn{1}{c}{ Cruzamento das variáveis } & $\mathbf{X}^{2}$ & $\boldsymbol{p}$ Valor \\
\hline Escolaridade x O que é uma RPPN e qual a sua finalidade? & 18,33 & $0,006^{* *}$ \\
Escolaridade x Percepção da importância das UCs para a conservação & 14,82 & $0,022^{*}$ \\
Escolaridade x Contribuições das RPPNs para a economia do país & 22,62 & 0,206 \\
\hline
\end{tabular}

**Altamente significativo $p-$ valor $<0,01 ; *$ Significativo $p-$ valor $<0,05$ Fonte: Dados da pesquisa (2016).

Em conformidade com os resultados das análises bivariadas é possível observar na Tabela 3 que o nível de escolaridade dos visitantes entrevistados apresentou associação altamente significativa quanto ao conhecimento sobre o "que é uma RPPN e qual a sua finalidade?" (X2 = 18,33 e p=0,006); a dependência das variáveis mostrou-se significativa para o quesito "Se você não tivesse visitado a RPPN, teria a mesma percepção sobre a importância das UCs para a conservação da natureza?" (X2 = 14,82 e $p=0,022$ ). Essa evidência está de acordo com Magalhães et al. (2010) e indica que quanto maior o nível de escolaridade, maior é o nível de conscientização quanto à importância da gestão da Unidade de Conservação.

Contudo, o relacionamento do grau de escolaridade com o entendimento "De que forma as RPPNs contribuem para a economia do país?" não apresentou relação significativa $(X 2=22,62$ e $p=0,206)$. Nessa perspectiva, pode-se inferir que a população visitante ainda não disponibiliza de conhecimento amplo sobre os serviços que uma RPPN pode oferecer. Como exemplo, os pagamentos por serviços ambientais, nos quais as RPPNs têm se mostrado instrumentos viáveis para essa finalidade. Em vista disso, Medeiros e Young (2011) mencionam que o papel das Unidades de Conservação não é facilmente internalizado na economia nacional e essa questão decorre, ao menos em parte, da falta de informações sistematizadas que esclareçam a sociedade sobre a contribuição das UCs para o desenvolvimento econômico e social do país. Por isso, há uma compreensão de que as atividades aplicadas nas RPPNs necessitam obter respaldo para divulgar as estratégias de conservação realizadas e a importância dessas áreas para o setor econômico.

Assim, inferiu-se que a percepção ambiental representa uma ferramenta estratégica de investigação que possibilita uma melhor compreensão sobre o processo perceptivo dos visitantes em relação ao ambiente natural e suas funções essenciais.

\subsection{AÇÕES QUE REFLETEM INDICADORES DE SUSTENTABILIDADE AMBIENTAL}

Os indicadores de sustentabilidade ambiental permitem monitorar o estado atual e futuras soluções referentes às estratégias de conservação. Por esse motivo, as visitas às RPPNs Quinta do Sol e Vale do Bugio apontaram 12 parâmetros que refletiram em indicadores de sustentabilidade ambiental e suas respectivas ocorrências (Tabela 4). Para tanto, levou em consideração que o conceito de sustentabilidade se caracteriza holístico e, além de compor a dimensão ambiental, inclui diversas implicações sociais e também os fatores econômicos (COHEN et al., 2014). 
Os indicadores identificados nas RPPNs Quinta do Sol e Vale do Bugio foram dimensionados conforme a escala de níveis proposta por Marangon e colaboradores.

Tabela 4 - Parâmetros e ocorrências das ações que refletem em indicadores de sustentabilidade ambiental nas reservas Quinta do Sol e Vale do Bugio, em conformidade com a escala de níveis: Alto (A), Médio (M) e Baixo (B)

\begin{tabular}{ll|l|l|l|l|l|l}
\hline \multicolumn{2}{c}{ PARÂMETROS } & \multicolumn{3}{c}{ OCORRÊNCIAS } \\
\cline { 5 - 9 } & Quinta do Sol & \multicolumn{2}{c}{ Vale do Bugio } \\
\hline Cursos de capacitação relacionados à conservação ambiental & $\mathrm{A}$ & & & & $\mathrm{M}$ & \\
\hline Desenvolvimento de pesquisa científica & $\mathrm{A}$ & & & $\mathrm{A}$ & & \\
\hline Destinação adequada dos resíduos sólidos & $\mathrm{A}$ & & & $\mathrm{A}$ & & \\
\hline Estudos sobre diversidade da fauna e da flora & $\mathrm{A}$ & & & $\mathrm{A}$ & & \\
\hline Eventos e ações de Educação Ambiental & $\mathrm{A}$ & & & $\mathrm{A}$ & & \\
\hline $\begin{array}{l}\text { Produção de mudas para reflorestamento local e recuperação } \\
\text { de áreas degradadas }\end{array}$ & $\mathrm{A}$ & & & & & $\mathrm{B}$ \\
\hline Projetos de conservação & $\mathrm{A}$ & & & $\mathrm{A}$ & & \\
\hline $\begin{array}{l}\text { Projetos de integração de áreas de entorno com a Reserva e } \\
\text { conservação de teritórios sustentáveis }\end{array}$ & $\mathrm{A}$ & & & $\mathrm{A}$ & & & \\
\hline Projetos de turismo sustentável/ecoturismo & & & $\mathrm{M}$ & & $\mathrm{A}$ & & \\
\hline Recuperação de áreas degradadas & $\mathrm{A}$ & & & & & $\mathrm{B}$ \\
\hline Reaproveitamento da água & $\mathrm{A}$ & & & & $\mathrm{M}$ & \\
\hline Utilização de tecnologias de baixo impacto & & $\mathrm{M}$ & & & & $\mathrm{B}$ \\
\hline
\end{tabular}

Fonte: Dados da pesquisa, adaptado de Marangon et al. (2004); Marinelli; Maltez (2010).

Na compreensão de que os indicadores são variáveis usadas para descreverem as características de um sistema ou entidade que se inter-relacionam (COHEN et al., 2014), foi possível identificar nas ações que refletem em indicadores de sustentabilidade ambiental com níveis de escala alto (A), entre $66,67 \%$ a $100 \%$, a ocorrência de dez parâmetros para a RPPN Quinta do Sol e sete relacionados à RPPN Vale do Bugio.

Entre esses indicadores, seis parâmetros foram comuns para as duas reservas. Ao levar em consideração que os indicadores permitem uma avaliação sobre a realidade do grau de sustentabilidade ambiental (VAN BELLEN, 2006), os resultados apresentados na Tabela 4, com níveis de escala alto (A) refletem a forma de gestão dessas reservas, bem como o impacto positivo das estratégias de ações desenvolvidas em prol da conservação e seus aspectos sociais e ambientais.

Para os parâmetros com níveis de escala médio (M), entre $33,34 \%$ e $66,66 \%$, as duas reservas apresentaram a mesma proporção, com a indicação de que esses elementos podem ser aprimorados. Com relação aos parâmetros com níveis de escala baixo (B), entre 1\% e 33,33\%, a RPPN Vale do Bugio apresentou três indicadores. Vale esclarecer que a reserva possui uma área mais conservada e uma diversidade de vegetação nativa, sem a necessidade de produção de mudas para reflorestamento e não possui áreas degradadas destinadas à recuperação ambiental. Quanto ao parâmetro relacionado à utilização de tecnologias de baixo impacto, preconiza-se reflexão, pois além de propiciar vantagens econômicas e ambientais, pode constituir uma das bases para um modelo de gestão sustentável.

Do ponto de vista dos indicadores apresentados, recomenda-se que as diretrizes e estratégias aplicadas à sustentabilidade nessas áreas sejam frequentemente atualizadas, com o intuito de prover informações sobre a eficiência e eficácia da conservação ambiental nesses locais.

Ainda sobre a importância das estratégias de conservação desenvolvidas pelas RPPNs em favor da proteção dos ambientes naturais no país, citam-se alguns casos de sucesso e dentre estes, vale 
destacar as iniciativas das RPPNs: Sesc Pantanal, no Mato Grosso; Reserva Natural Salto Morato, Paraná; Santuário de Vida Silvestre Vagafogo, Goiás; e da RPPN Buraco das Araras, no Mato Grosso do Sul, que alia o ecoturismo à conservação da arara-vermelha (Ara chloropterus).

Nesse sentido, a Confederação Nacional de Reservas Particulares do Patrimônio Natural aponta a necessidade de valorização das RPPNs pelos órgãos públicos e sociedade em geral, uma vez que as RPPNs revestem-se de grande importância para a conservação da biodiversidade, ampliação e conectividade das paisagens, e foco nas relações socioambientais em todas as regiões brasileiras.

\section{CONSIDERAÇÕES FINAIS}

Ao analisar as estratégias relacionadas à conservação ambiental nas RPPNs Quinta do Sol e Vale do Bugio constatou-se que há uma maior preocupação dos visitantes sobre a conservação ambiental e que essa temática tem se tornado cada vez mais crescente.

Verificou-se ainda que o ecoturismo e a pesquisa científica se configuraram como as principais estratégias utilizadas pelas RPPNs para a motivação das visitas às Áreas Protegidas e representaram fatores importantes que beneficiam a viabilidade de ações de conservação.

Além disso, os indicadores se destacaram como uma ferramenta para o diagnóstico ambiental das duas reservas e podem servir de referência para novos estudos e direcionarem os gestores na realização e manutenção de práticas sustentáveis.

As RPPNs Quinta do Sol e Vale do Bugio se destacaram pelo sucesso das estratégias relacionadas à conservação ambiental que ocorrem devido à realização de boas práticas de sustentabilidade. As estratégias utilizadas por essas reservas contribuem significativamente para a expansão das áreas protegidas em regiões importantes, como é o caso da Serra de Maracaju, área prioritária para conservação da biodiversidade.

Apesar das dificuldades que ocorrem, tanto na criação como na sustentação desses espaços, são notórios os esforços dos gestores em estabelecer melhorias nas estratégias que direcionam a gestão dessas reservas naturais. No entanto, é fundamental o apoio contínuo do poder público, associações e instituições às ações de conservação empregadas nas RPPNs, a fim de possibilitar a proteção ambiental e o desenvolvimento social e econômico.

Portanto, as RPPNs não apenas podem favorecer a proteção ambiental, mas também devem assumir o compromisso de oportunizar transformações sociais por meio da educação ambiental e de atividades direcionadas para o desenvolvimento, que vão além dos limites da reserva. Por esse motivo, sugerese que o processo de difusão e o desenvolvimento de ações para a conservação ambiental ocorram continuadamente, a fim de gerar e manter a sustentabilidade no presente e em longo prazo, bem como aproximar as pessoas da natureza, qualificar essas interações e engajá-las em propostas de conservação ambiental.

\section{AGRADECIMENTOS}

Aos proprietários e gestores das RPPNs Estância Ambiental Quinta do Sol e Reserva Ecológica Vale do Bugio; à Associação de RPPNs de Mato Grosso do Sul; ao Instituto Mamede de Pesquisa Ambiental e Ecoturismo; e à Coordenação de Aperfeiçoamento de Pessoal de Nível Superior (Capes).

\section{REFERÊNCIAS}

AB'SABER, A. N. Os domínios de natureza no Brasil: potencialidades paisagísticas. 7. ed. São Paulo: Ateliê Editorial, 2012. 
ALVES, T.; BOOCK, J.; SOUSA, L. M. RPPN (Private Natural Heritage Reserve). History of Private Land Conservation in the State of Mato Grosso of Sul. Campo Grande: REPAMS e WWF-Brasil, 2016.

BRASIL. Lei no 9.985, de 18 de julho de 2000. Institui o Sistema Nacional de Unidades de Conservação da Natureza. Diário Oficial da República Federativa do Brasil, Brasília, DF, 19 de jul. de 2000.

BRASIL. Diretrizes para visitação em Unidades de Conservação. Brasília: MMA, 2008.

BURSZTYN, M. Meio ambiente e interdisciplinaridade: desafios ao mundo acadêmico. Desenvolvimento e Meio Ambiente, n. 10, p. 67-76, 2004.

CARDOSO, M. R. C.; CARDOSO, G. C. C.; BRITO, J. M. B. S. Economia e planejamento do ecoturismo: estudo de caso no Cerrado brasileiro. Sustentabilidade em Debate, v. 6, n. 3, p. 100-115, 2015.

CHAPE, S. et al. Measuring the extent and effectiveness of protected areas as an indicator for meeting global biodiversity targets. Biological Sciences, v. 360, p. 443-455, 2005.

COHEN, S. et al. The Growth of Sustainability Metrics. Earth Institute, p. 1-16, 2014.

COSTANZA, R. et al. The value of the world's ecosystem services and natural capital. Nature, 387, 253-260, 1997.

CRESWELL, J. W.; PLANO CLARK, V. L. Designing and conducting mixed methods research. 2nd ed. Los Angeles: SAGE Publications, 2011.

CROUZEILLES, R. et al. Increasing strict protection through protected areas on Brazilian private lands. Environmental Conservation, v. 40, n. 3, p. 209-210. 2012.

DOUROJEANNI, M. J.; PÁDUA, M. T. J. Arcas à deriva: unidades de conservação do Brasil. Rio de Janeiro: Technical Books, 2013.

ELBAKIDZE, M. et al. Protected Area as an Indicator of Ecological Sustainability? A Century of Development in Europe's Boreal Forest. AMBIO, v. 42, p. 201-214, 2013.

FILETTO, F.; MACEDO, R. L. G. Desenvolvimento de indicadores de sustentabilidade para o Ecoturismo em Unidades de Conservação. Revista Brasileira de Ecoturismo, v. 8, n. 1, p. 11-30, 2015.

GODINHO, R. S.; MOTA, M. J. P. Desafios da Convenção sobre a diversidade biológica. Revista de Direito da Cidade, v. 5, n. 2, p. 106-136, 2013.

GONÇALVES, N. M.; HOEFFEL, J. L. M. Percepção Ambiental sobre Unidades de Conservação: os conflitos em torno do Parque Estadual de Itapetinga - SP. Revista VITAS - Visões Transdisciplinares sobre Ambiente e Sociedade, n. 3, p. 2012.

INSTITUTO BRASILEIRO DE GEOGRAFIA E ESTATÍSTICA. Estados. Disponível em: <http://www.ibge.gov.br/ estadosat/perfil.php?sigla=ms $>$. Acesso em: 10 out. 2016.

KAMAL, S.; GRODZINSKA-JURCZAK, M.; BROWN, G. Conservation on private land: a review of global strategies with a proposed classification system. Journal of Environmental Planning and Management, v. 58, n. 4, p. 576597, 2015

KEUROGHLIAN, A.; SANTOS, M. C. A.; EATON, D. P. The effects of deforestation on white-lipped peccary (Tayassu pecari) home range in the southern Pantanal. Mammalia, p. 1-7, 2014.

KLEIN, F. M. et al. Educação ambiental e o ecoturismo na Serra da Bodoquena em Mato Grosso do Sul. Sociedade \& Natureza, n. 2, p. 311-321, 2011.

LE SAOUT, S. et al. Protected areas and effective biodiversity conservation. Science, v. 342, p. 803-805, 2013.

LEWINSOHN, T. M.; PRADO, P. I. Quantas espécies há no Brasil? Revista Megadiversidade, v. 1, n. 1, p. 36-42, 2005. 
LUCENA, M. M. A.; FREIRE, E. M. X. Percepção Ambiental sobre uma Reserva Particular do Patrimônio Natural (RPPN), pela Comunidade Rural do Entorno, Semiárido brasileiro. Educação Ambiental em Ação, n. 35, p. 1-16, 2011.

MAGALHÃES, H.; BONONI, V. L. R.; MERCANTE, M. A. Participação da sociedade civil na gestão de unidades de conservação e seus efeitos na melhoria da qualidade ambiental da Região Sudeste do Estado do Mato Grosso do Sul. Acta Scientarum, v. 32, n. 2, p. 183-192, 2010.

MAGANHOTTO, R. F. et al. Unidades de Conservação: limitações e contribuições para a conservação da natureza. Sustentabilidade em Debate, v. 5, n. 3, p. 203-221, 2014.

MARANGON, M. et al. Indicadores de sustentabilidade como instrumento para avaliação de comunidades em crise: aplicação à comunidade de Serra Negra. Revista Educação \& Tecnologia, v. 8, p. 143-161, 2004.

MARINELLI, C. E.; MALTEZ, H. M. Indicadores socioambientais de Unidades de Conservação. Documento síntese: workshop. Instituto Socioambiental, Brasília, DF, 2010.

MATHEUS, F. S.; RAIMUNDO, S. Public use and Ecotourism Policies in Brazilian and Canadian Protected Areas. Etudes caribéennes, n. 33-34, 2016.

MEDEIROS, R.; YOUNG, C. E. F. Contribuição das Unidades de Conservação brasileiras para a economia nacional. Brasília: UNEP - WCMC, 2011.

MOLDAN, B.; JANOUŠKOVÁ, S.; HÁK, T. How to understand and measure environmental sustainability: indicators and targets. Ecological Indicators, v. 17, p. 4-13, 2012.

MORAES, M. C. P.; MELLO, K.; TOPPA, R. H. Gestão integrada em unidades de conservação: estudo de caso do Parque Estadual de Porto Ferreira. Revista Brasileira de Ciências Ambientais, n. 33, p. 45-59, 2014.

MORELLI, J. Environmental Sustainability: a definition for environmental professionals. Journal of Environmental Sustainability, v. 1, n. 1, p. 1-10, 2011.

MYERS, N. et al. Biodiversity hotspots for conservation priorities. Nature, v. 403, p. 453-458, 2000.

NEIMAN, Z. Ecoturismo e educação ambiental em unidades de conservação: a importância da experiência dirigida. In: COSTA, N. M. C.; NEIMAN, Z.; COSTA, V. C. (Org.) Pelas trilhas do ecoturismo. Rima, 2008.

OLIVEIRA, A. P. G. et al. Uso de geotecnologias para o estabelecimento de áreas para corredores de biodiversidade. Revista Árvore, v. 39, n. 4, p. 595-602, 2015.

PEGAS, F. V.; CASTLEY, J. G. Ecotourism as a conservation tool and its adoption by private protected areas in Brazil. Journal of Sustainable Tourism, v. 22, n. 4, p. 604-625, 2014.

PELLIN, A.; RANIERI, V. E. L. Motivações para o estabelecimento de RPPNs e análise dos incentivos para sua criação e gestão no Mato Grosso do Sul. Natureza \& Conservação, v. 7, n. 2, p. 72-81, 2009.

SAKELLARI, M.; SKANAVIS, C. Sustainable tourism development: environmental education as a tool to fill the gap between theory and practice. International Journal of Environment and Sustainable Development, v. 12. n. 4, p. 313-323, 2013.

SCHERL, L. M. et al. As áreas protegidas podem contribuir para a redução da pobreza? Oportunidades e limitações. Gland, Suíça e Cambridge, Reino Unido: IUCN, 2006.

VAN BELLEN, H. M. Indicadores de Sustentabilidade: uma análise comparativa. Rio de Janeiro: Editora FGV, 2006.

WATSON, J. E. M. et al. The performance and potential of protected areas. Nature, v. 515, p. 19-25, 2014.

WILSON, E. O. Biophilia: the human bond with other species. Cambridge: Harvard University Press, 1984. 